\title{
Estimation Accuracy of Exponential Distribution Parameters
}

\author{
Muhammad Zahid Rashid \\ College of Statistical and Actuarial Sciences \\ University of the Punjab, Q.A. Campus \\ Lahore, Pakistan \\ zahidcsas@gmail.com \\ Ahmad Saeed Akhter \\ College of Statistical and Actuarial Sciences \\ University of the Punjab, Q.A. Campus \\ Lahore, Pakistan \\ akhtar@stat.pu.edu.pk
}

\begin{abstract}
The exponential distribution is commonly used to model the behavior of units that have a constant failure rate. The two-parameter exponential distribution provides a simple but nevertheless useful model for the analysis of lifetimes, especially when investigating reliability of technical equipment.

This paper is concerned with estimation of parameters of the two parameter (location and scale) exponential distribution. We used the least squares method (LSM), relative least squares method (RELS), ridge regression method (RR), moment estimators (ME), modified moment estimators (MME), maximum likelihood estimators (MLE) and modified maximum likelihood estimators (MMLE). We used the mean square error MSE, and total deviation TD, as measurement for the comparison between these methods. We determined the best method for estimation using different values for the parameters and different sample sizes.
\end{abstract}

Keywords: Exponential distribution, Parameter estimation, Least squares method, Relative least squares, Ridge regression, Moment estimators, Modified moment estimators, Maximum likelihood estimators, Modified maximum likelihood estimators, Total deviation, Mean square error.

\section{Introduction}

Exponential distribution plays an important part in the field of reliability engineering and life testing. The exponential distribution would be an appropriate choice for an experimenter, if failure rate appears to be more or less constant. "This is a distribution of the time to an event when the probability of the event occurring in the next small time interval does not vary through time. It is used in the theory of waiting lines or queues, which are found in many situations: from the gates at the entrance to toll roads through the time taken for an answer to a telephone enquiry, to the time taken for an ambulance to arrive at the scene of an accident" Evans et al; (2000). Exponential distribution is a special case of the Weibull distribution where $\beta=1$.

The exponential distribution can be found with one or two parameters; scale and location parameters. The values of these parameters must be more than zero except for the location parameter which can be greater than or equal to zero. 
The probability density function of two parameter exponential distribution is given by

$$
f\left(t_{i}, \gamma, \beta\right)=\frac{1}{\beta} \exp \left[-\frac{\left(t_{i}-\gamma\right)}{\beta}\right] t \geq \gamma ; \beta>0, \gamma<t<\infty
$$

The parameters $\gamma$ and $\beta$ are interpreted as measure of guarantee and failure rate respectively.

Maguire, Pearson and Wynn (1952) studied mine accidents and showed that time intervals between industrial accidents follow exponential distribution. Cohen and Helm (1973) used (BLUE), (MLE), (ME), (MVUE) and MME to estimate the parameters of the exponential distribution. Peter (1974) used robust M-estimation method for the scale parameter, with application to the exponential distribution. Cohen and Whitten (1982) used the moment and modified moment estimators for the Weibull distribution. Samia and Mohamed (1993) used five modifications of moments to estimate the parameters of the Pareto distribution. Lalitha and Anand (1996) used modified maximum likelihood to estimate the scale parameter of the Rayleigh distribution. Kang and Young (1997) estimated the parameters of a Pareto distribution by jackknife and bootstrap methods. Razali et al. (2009) studied the estimation accuracy of three parameter Weibull distribution.

In this paper, we use the least squares method, relative least squares, ridge regression, moment and modified moment estimators, maximum and modified maximum likelihood estimators to estimate the two parameter of the exponential distribution. The present paper introduces third modified moment estimators and first modified maximum likelihood estimators. Also, we compared between these methods using two parameter exponential distributions to find the most accurate method.

\section{Methodology}

\section{Least squares method (LSM)}

For the estimation of probability distribution parameters, the least squares method (LSM) is extensively used in reliability engineering and mathematics problems.

The cumulative distribution function of (1) is given by

$$
\begin{aligned}
& F\left(t_{i}\right)=1-\exp \left[-\frac{\left(t_{i}-\gamma\right)}{\beta}\right] \\
& \Rightarrow \exp \left[-\frac{\left(t_{i}-\gamma\right)}{\beta}\right]=1-F\left(t_{i}\right)
\end{aligned}
$$


To get a linear relation between the two parameters taking the natural logarithm of above equation as follows

$$
\frac{\left(t_{i}-\gamma\right)}{\beta}=\ln \left[1-F\left(t_{i}\right)\right.
$$

After simplification, we get

$$
t_{i}=\gamma+\beta\left[-\ln \left(1-F\left(t_{i}\right)\right]\right.
$$

The last equation can be represented by

$$
y_{i}=a+b x_{i}
$$

Where $y_{i}=t_{i}, a=\gamma, \quad b=\beta, \quad x_{i}=\left[-\ln \left(1-F\left(t_{i}\right)\right)\right] i=1,2, \ldots \ldots \ldots \ldots n$ and $n$ is the sample size.

Let $t_{1}, t_{2}, t_{3}, \ldots \ldots . n$ be a random sample of $t_{i}$ and $F\left(t_{i}\right)$ is estimated and replaced by the median rank method as follows:

$$
F\left(t_{i}\right)=\frac{(i-0.3)}{(n+0.4)} \quad t_{i}, i=1,2, \ldots \ldots, n \quad\left(t_{1}<t_{2}<t_{3} \ldots<t_{n}\right)
$$

Because $F\left(t_{i}\right)$ of the mean rank method

$$
F\left(t_{i}\right)=\frac{i}{(n+1)}
$$

May be a larger value for smaller $i$ and a smaller value for larger $i$.

Thus, equation (2) is a linear equation and is expressed as

$$
y_{i}=a+b x_{i}
$$

To compute $a$ and $b$ by simple linear regression we proceed as follows.

Let

$$
S(a, b)=\sum_{i=1}^{n}\left(Y_{i}-a-b X_{i}\right)^{2}
$$

Differentiating $S$ w.r.t $a$ and $b$ then equate to zero, we obtain the following two normal equations

$$
\begin{gathered}
\sum_{i=1}^{n} Y_{i}=n a+b \sum_{i=1}^{n} X_{i} \\
\sum_{i=1}^{n} X_{i} Y_{i}=a \sum_{i=1}^{n} X_{i}+b \sum_{i=1}^{n} X_{i}^{2}
\end{gathered}
$$


Solving the above two equations for $a$ and $b$, we obtain the least square estimates (LSE) of $a$ and $b$ as:

$$
\begin{aligned}
& \hat{a}=\frac{\sum_{i=1}^{n}\left[-\ln \left(1-F\left(t_{i}\right)\right)\right] \sum_{i=1}^{n} t_{i}\left[-\ln \left(1-F\left(t_{i}\right)\right)\right]-\sum_{i=1}^{n} t_{i} \sum_{i=1}^{n}\left[-\ln \left(1-F\left(t_{i}\right)\right)\right]^{2}}{\left[\sum_{i=1}^{n}\left(-\ln \left(1-F\left(t_{i}\right)\right)\right)\right]^{2}-n \sum_{i=1}^{n}\left[-\ln \left(1-F\left(t_{i}\right)\right)\right]^{2}} \\
& \hat{b}=\frac{\sum_{i=1}^{n} t_{i}\left[-\ln \left(1-F\left(t_{i}\right)\right)\right]-n \sum_{i=1}^{n} t_{i}\left[-\ln \left(1-F\left(t_{i}\right)\right)\right]}{\left[\sum_{i=1}^{n}\left(-\ln \left(1-F\left(t_{i}\right)\right)\right)\right]^{2}-n \sum_{i=1}^{n}\left[-\ln \left(1-F\left(t_{i}\right)\right)\right]^{2}}
\end{aligned}
$$

\section{Relative Least squares Method (RELS)}

The relative least squares estimators of $a$ and $b$ can be obtained by minimizing the sum of squares of the relative residuals, Pablo and Bruce (1992), w.r.t. a and $b$ as follows

$$
\begin{aligned}
& S=\sum_{i=1}^{n}\left(\frac{Y_{i}-a-b X_{i}}{Y_{i}}\right)^{2} \\
& S=\sum_{i=1}^{n}\left(1-a w_{i}-b z_{i}\right)^{2}
\end{aligned}
$$

Differentiating w.r.t, $a$ and $b$ then equate to zero

$$
\begin{gathered}
\sum_{i=1}^{n} w_{i}=a \sum_{i=1}^{n} w_{i}^{2}+b \sum_{i=1}^{n} w_{i} z_{i} \\
\sum_{i=1}^{n} z_{i}=a \sum_{i=1}^{n} w_{i} z_{i}+b \sum_{i=1}^{n} z_{i}^{2}
\end{gathered}
$$

After simplification, we get

$$
\begin{aligned}
& \hat{a}=\frac{\sum_{i=1}^{n} w_{i} z_{i} \sum_{i=1}^{n} z_{i}-\sum_{i=1}^{n} w_{i} \sum_{i=1}^{n} z_{i}^{2}}{\left(\sum_{i=1}^{n} w_{i} z_{i}\right)^{2}-\sum_{i=1}^{n} w_{i}^{2} \sum_{i=1}^{n} z_{i}^{2}} \\
& \hat{b}=\frac{\sum_{i=1}^{n} w_{i} z_{i} \sum_{i=1}^{n} w_{i}-\sum_{i=1}^{n} w_{i}^{2} \sum_{i=1}^{n} z_{i}^{2}}{\left(\sum_{i=1}^{n} w_{i} z_{i}\right)^{2}-\sum_{i=1}^{n} w_{i}^{2} \sum_{i=1}^{n} z_{i}^{2}}
\end{aligned}
$$

Where $w_{i}=\frac{1}{t_{i}}$ and $z_{i}=\frac{\left[-\ln \left(1-F\left(t_{i}\right)\right)\right]}{t_{i}}$

\section{Ridge regression method (RR):}

The ridge regression ( $R R$ ) estimates of $A$ and $B$ can be obtained by minimizing the error sum of squares for the model

$$
y_{i}=a+b x_{i}
$$


Subject to the single constraint that $a^{2}+b^{2}=\phi$ where $\phi$ is a finite positive constant.

The method of Lagrange's multiplier requires the differentiation of

$$
L=\sum_{i=1}^{n}\left(y_{i}-a-b x_{i}\right)^{2}+\lambda\left(a^{2}+b^{2}-\phi\right)
$$

W.r.t a and b. when these derivatives are equated to zero, we obtain the following two equations

$$
\begin{aligned}
& \sum_{i=1}^{n} y_{i}=(n+\lambda) a+b \sum_{i=1}^{n} x_{i} \\
& \sum_{i=1}^{n} x_{i} y_{i}=a \sum_{i=1}^{n} x_{i}+b\left(\lambda+\sum_{i=1}^{n} x^{2}{ }_{i}\right)
\end{aligned}
$$

Solving above two equations for $a$ and $b$ we get

$$
\begin{gathered}
\hat{a}=\frac{\left(\sum_{i=1}^{n} x_{i}\right)\left(\sum_{i=1}^{n} x_{i} y_{i}\right)-\sum_{i=1}^{n} y_{i}\left(\lambda+\sum_{i=1}^{n} x^{2}{ }_{i}\right)}{\left(\sum_{i=1}^{n} x_{i}\right)^{2}-(n+\lambda)\left(\lambda+\sum_{i=1}^{n} x^{2}{ }_{i}\right)} \\
\hat{b}=\frac{\left(\sum_{i=1}^{n} x_{i}\right)\left(\sum_{i=1}^{n} y_{i}\right)-(n+\lambda) \sum_{i=1}^{n} x_{i} y_{i}}{\left(\sum_{i=1}^{n} x_{i}\right)^{2}-(n+\lambda)\left(\lambda+\sum_{i=1}^{n} x^{2}{ }_{i}\right)}
\end{gathered}
$$

For two parameter exponential distribution we know that $y_{i}=t_{i}, a=\gamma, b=\beta$

$$
x_{i}=\left[-\ln \left(1-F\left(t_{i}\right)\right)\right] \quad i=1,2, \ldots \ldots \ldots \ldots . n
$$

$$
\begin{gathered}
\hat{\gamma}=\frac{\sum_{i=1}^{n}\left[-\ln \left(1-F\left(t_{i}\right)\right)\right] \sum_{i=1}^{n} t_{i}\left[-\ln \left(1-F\left(t_{i}\right)\right)\right]-\sum_{i=1}^{n} t_{i}\left(\lambda+\sum_{i=1}^{n}\left[-\ln \left(1-F\left(t_{i}\right)\right)\right]^{2}\right)}{\left[\sum_{i=1}^{n}\left(-\ln \left(1-F\left(t_{i}\right)\right)\right)\right]^{2}-(n+\lambda)\left(\lambda+\sum_{i=1}^{n}\left[-\ln \left(1-F\left(t_{i}\right)\right)\right]^{2}\right)} \\
\hat{\beta}=\frac{\sum_{i=1}^{n} t_{i} \sum_{i=1}^{n}\left(-\ln \left(1-F\left(t_{i}\right)\right)\right)-(n+\lambda) \sum_{i=1}^{n} t_{i}\left(-\ln \left(1-F\left(t_{i}\right)\right)\right)}{\left[\sum_{i=1}^{n}\left(-\ln \left(1-F\left(t_{i}\right)\right)\right)\right]^{2}-(n+\lambda)\left(\lambda+\sum_{i=1}^{n}\left[-\ln \left(1-F\left(t_{i}\right)\right)\right]^{2}\right.}
\end{gathered}
$$

Where $0<\lambda<1$ the ridge coefficient is the readers may see Ronald and Raymond (1978) if $\lambda=0$ we obtain the least square estimates. 


\section{Moment Estimators (ME)}

For the two parameter exponential distribution, the first two moments of exponential distribution are given by

$$
\begin{aligned}
& \mu_{1}=E(x)=\gamma+\beta \\
& \mu_{2}^{\prime}=E\left(x^{2}\right)=\gamma^{2}+2 \beta^{2}+2 \gamma \beta
\end{aligned}
$$

The variance of $\mathrm{x}$ is given by

$$
\begin{aligned}
\operatorname{var}(x) & =\mu_{2}^{\prime}-\mu^{2}{ }_{1} \\
& =\gamma^{2}+2 \beta^{2}+2 \gamma \beta-(\gamma+\beta) \\
& =\beta^{2}
\end{aligned}
$$

By equating the first two population moments with sample moments

$$
\begin{aligned}
& \bar{x}=\frac{\left(\sum_{i=1}^{n} x_{i}\right)}{n} \\
\Rightarrow \quad & m_{1}=\bar{x} \\
\text { and } \quad s^{2} & =\frac{\sum_{i=1}^{n}\left(x_{i}-\bar{x}\right)^{2}}{n-1} \\
m_{1} & =\gamma+\beta \\
s^{2} & =\beta^{2}
\end{aligned}
$$

From above equation the estimate of $\beta$ is given by $\hat{\beta}=s$.

By substitute about $\hat{\beta}$ into (15) the estimate of $\gamma$ is given by $\hat{\gamma}=m_{1}-s$.

\section{First Modified Moment Estimators (MME-I)}

In this modification of the moment estimators, the second moment of two parameter exponential distribution is replaced by

$$
E\left[F\left(t_{(1)}\right)\right]=F\left(t_{(1)}\right)
$$

Where $t_{(1)}$ is the first order statistic

$$
\begin{aligned}
& \frac{1}{n+1}=1-\exp \left[-\frac{\left(t_{(1)}-\gamma\right)}{\beta}\right] \\
& -\frac{\left(t_{(1)}-\gamma\right)}{\beta}=\ln \left(\frac{n}{n+1}\right)
\end{aligned}
$$


We know that the first sample standard moment

$$
\begin{aligned}
& m_{1}=\gamma+\beta \\
& \gamma=m_{1}-\beta
\end{aligned}
$$

The solution of (18) and (17) is given the estimate of $\beta$ as

$$
\begin{aligned}
& \hat{\beta}=\frac{m_{1}-t_{(1)}}{1+\ln \left(\frac{n}{n+1}\right)} \\
& \hat{\beta}=Z\left(m_{1}-t_{(1)}\right)
\end{aligned}
$$

Where

$$
Z=\frac{1}{1+\ln \left(\frac{n}{n+1}\right)}
$$

From (18) and (19) we can obtain the estimate of $\gamma$ as

$$
\begin{aligned}
& \hat{\gamma}=m_{1}-Z\left(m_{1}-t_{(1)}\right) \\
& \hat{\gamma}=(1-Z) m_{1}+Z\left(t_{(1)}\right)
\end{aligned}
$$

\section{Second Modified Moment Estimators (MME-II)}

In this case, the second standard moment of two parameter exponential distribution is replaced by

$$
E\left(t_{(1)}\right)=t_{(1)}
$$

That is

$$
\gamma+\frac{\beta}{n}=t_{(1)}
$$

Solution of (18) and (21) is given the estimate of

$$
\hat{\beta}=\frac{n\left(m_{1}-t_{(1)}\right)}{(n-1)}
$$

From equations (21) and (22) we can obtain the estimate of $\gamma$ as

$$
\hat{\gamma}=\frac{n\left(t_{(1)}-m_{1}\right)}{(n-1)}
$$

\section{Third Modified Moment estimators (MME-III)}


In this modification the second moment is replaced by $M_{e t}=t_{m e}$, where $\mathrm{Me}_{\mathrm{t}}$ is the population median and $t_{m e}$ is the sample median.

The median of two parameter exponential distribution is given by

$$
\begin{aligned}
& \frac{1}{\beta} \int_{0}^{a} \exp \left[-\frac{\left(t_{i}-\gamma\right)}{\beta}\right] d t=\frac{1}{2} \\
& a=\gamma+\beta(\ln 2)
\end{aligned}
$$

Thus, we have

$$
\gamma+\beta(\ln 2)=t_{m e}
$$

From equation (18) and (24) we obtain

$$
\hat{\beta}=\frac{\left(m_{1}-t_{m e}\right)}{(1-\ln 2)}
$$

From equation (24) and (25), we obtain

$$
\hat{\gamma}=\frac{\left(t_{m e}-m_{1}(\ln 2)\right)}{(1-\ln 2)}
$$

\section{Maximum likelihood estimator (MLE)}

The likelihood function can be shown as follows,

$$
L(\gamma, \beta)=\prod^{n}{ }_{i=1} f\left(t_{i,}, \beta\right)
$$

The maximum likelihood estimator MLE of the parameter is the value of the parameter that maximizes $L$ and MLE for 2-parameter exponential distribution can be obtained by solving the equations resulting from setting the two partial derivatives of $\mathrm{L}(\gamma, \beta)$ to zero;

$$
\begin{aligned}
& L(\gamma, \beta)=\prod_{i=1}^{n} \frac{1}{\beta} \exp \left[-\frac{\left(t_{i}-\gamma\right)}{\beta}\right] \\
& \frac{\partial \ln L}{\partial \gamma}=\frac{n}{\beta}=0
\end{aligned}
$$

It can be seen that $L\left(\gamma, t_{m}\right)$ is monotonically increasing with $t_{m}$ i.e. the greater the value of $t_{m}$ the greater the value of likelihood function.

Hence since $\mathrm{t} \geq \mathrm{t}_{\mathrm{m}}$ we conclude

$$
\begin{aligned}
& \hat{\gamma}=\min \left(t_{i}\right)=y_{1} \\
& \frac{\partial \ln L}{\partial \gamma}=-\frac{n}{\beta}+\frac{\sum_{i=1}^{n}\left(t_{i}-\gamma\right)}{\beta^{2}}
\end{aligned}
$$




$$
\begin{aligned}
& \beta=\frac{\sum_{i=1}^{n}\left(t_{i}-\gamma\right)}{n}=\bar{t}-\gamma \\
& \hat{\beta}=\bar{t}-y_{1}
\end{aligned}
$$

\section{First Modified Maximum Likelihood estimator}

The first equation of MLM $\hat{y}=y_{1}$ is replaced by

$$
\begin{aligned}
& E\left(Y_{(1)}\right)=Y_{(1)} \\
& \gamma+\frac{\beta}{n}=y_{(1)}
\end{aligned}
$$

By solving equation (32) and (31) simultaneously, we obtain the parameter estimates

$$
\hat{\gamma}=\frac{(n+1) y_{(1)}-\bar{t}}{n}
$$

\section{Goodness of Fit Analysis}

Some methods of goodness of fit analysis are employed here. Mean square error MSE and total deviation TD are two measurements that give an indication of the accuracy of parameter estimation. AL-Fawzan (2000) referred to the use of the procedure of MSE and TD.

\section{a. Mean Square Errors (MSE)}

The MSE can be calculated as below

$$
\begin{aligned}
& M S E=\sum_{i=1}^{n}\left\{\hat{F}\left(t_{i}\right)-F\left(t_{i}\right)\right\}^{2} \\
& \text { Where } \hat{F}\left(t_{i}\right)=1-\exp \left[-\frac{\left(t_{i}-\hat{\gamma}\right)}{\hat{\beta}}\right]
\end{aligned}
$$

is the value of the cumulative distribution function of the two parameter exponential distribution using the estimated parameters, and

$$
F\left(t_{i}\right)=1-\exp \left[-\frac{\left(t_{i}-\gamma\right)}{\beta}\right]
$$

\section{b. Total Derivation (TD)}

The total derivation TD, calculated for each method is as follows

$$
T D=\left|\frac{\hat{\gamma}-\gamma}{\gamma}\right|+\left|\frac{\hat{\beta}-\beta}{\beta}\right|
$$

Where $\gamma$ and $\beta$ are the known parameters, and $\hat{\gamma}$ and $\hat{\beta}$ are the estimated parameters by any method. 


\section{Application}

A simulation study is used in order to compare the performance of the proposed estimation methods. According to limitations of the computer time, we carry out this comparison taking the sample sizes as $n=20,40,60,80$ and 100 with pairs of $(\gamma, \beta)=(1,1),(1,2),(2,3)$ we generated random samples of different sizes by observing that if $U$ is uniform $(0,1)$, then

$$
T=\gamma+\beta[-\ln (1-u)] \text { is exponential of }(\gamma, \beta)
$$

Such generated data have been used to obtain estimates of the unknown parameters. The results obtained from parameter estimation of the 2-parameter exponential distribution using different sample sizes and different values of parameters with mean square error MSE and total deviation TD, are given in tables (1), (2), (3), (4) and (5).

Table 1: The estimates for $n=20$

\begin{tabular}{|c|c|c|c|c|c|c|}
\hline \multirow{2}{*}{ Method } & \multicolumn{2}{|c|}{ True Values } & \multicolumn{2}{|c|}{ Estimated Values } & \multirow{2}{*}{ MSE } & \multirow{2}{*}{ TD } \\
\hline & $\gamma$ & $\beta$ & $\hat{\gamma}$ & $\hat{\beta}$ & & \\
\hline \multirow{3}{*}{ LSM } & 1 & 1 & 0.999997763 & 0.9999864 & $3.83633 \times 10^{-11}$ & 0.0000158 \\
\hline & 1 & 2 & 1.00002381 & 1.99998 & $6.45067 \times 10^{-10}$ & 0.0000333 \\
\hline & 2 & 3 & 2.00000623 & 3.00000825 & $6.0299 \times 10^{-11}$ & 0.0000059 \\
\hline \multirow{3}{*}{ RELS } & 1 & 1 & 0.95797 & 1.0000018 & 0.0114253 & 0.0420285 \\
\hline & 1 & 2 & 0.9999 & 2.18104 & 0.0097119 & 0.0905306 \\
\hline & 2 & 3 & 1.99999 & 2.999998 & $1.64 \times 10^{-12}$ & 0.000001 \\
\hline \multirow{3}{*}{$\mathrm{RR}$} & 1 & 1 & 0.973875 & 1.00162 & 0.00432129 & 0.027745 \\
\hline & 1 & 2 & 0.999202 & 1.97792 & 0.0001753 & 0.011838 \\
\hline & 2 & 3 & 1.97308 & 2.97954 & 0.0008347 & 0.02028 \\
\hline \multirow{3}{*}{ ME } & 1 & 1 & 1.06899 & 1.02620 & 0.0408251 & 0.09519 \\
\hline & 1 & 2 & 1.13799 & 2.0524 & 0.0408306 & 0.16419 \\
\hline & 2 & 3 & 2.206977 & 3.0786 & 0.0408276 & 0.129689 \\
\hline \multirow{3}{*}{ (MME-I) } & 1 & 1 & 0.944557 & 1.15063 & 0.0150322 & 0.0206073 \\
\hline & 1 & 2 & 0.88912 & 2.30126 & 0.0150319 & 0.26151 \\
\hline & 2 & 3 & 1.83367 & 3.45190 & 0.0150327 & 0.233798 \\
\hline \multirow[t]{3}{*}{ (MME-II) } & 1 & 1 & 0.943095 & 1.15209 & 0.0153931 & 0.208995 \\
\hline & 1 & 2 & 0.88618 & 2.3042 & 0.0153951 & 0.26592 \\
\hline & 2 & 3 & 1.82927 & 3.45630 & 0.0153951 & 0.237465 \\
\hline \multirow{3}{*}{ (MME-III) } & 1 & 1 & 1.00664 & 1.08855 & 0.0596031 & 0.09519 \\
\hline & 1 & 2 & 1.1771021 & 2.0132779 & 0.0596043 & 0.183741 \\
\hline & 2 & 3 & 2.26565 & 3.01993 & 0.0596040 & 0.139468 \\
\hline \multirow{3}{*}{ MLE } & 1 & 1 & 1.00070 & 1.09449 & 0.0107806 & 0.09519 \\
\hline & 1 & 2 & 1.00139 & 2.18899 & 0.0107800 & 0.095885 \\
\hline & 2 & 3 & 2.00209 & 3.283485 & 0.0107805 & 0.09554 \\
\hline \multirow{3}{*}{ MMLE-I } & 1 & 1 & 0.9459755 & 1.09449 & 0.0098298 & 0.148515 \\
\hline & 1 & 2 & 0.8919405 & 2.18899 & 0.0098315 & 0.202555 \\
\hline & 2 & 3 & 1.837915 & 3.283485 & 0.0098310 & 0.175538 \\
\hline
\end{tabular}


Estimation Accuracy of Exponential Distribution Parameters

Table 2: The estimates for $n=40$

\begin{tabular}{|c|c|c|c|c|c|c|}
\hline \multirow{2}{*}{ Method } & \multicolumn{2}{|c|}{ True Values } & \multicolumn{2}{|c|}{ Estimated Values } & \multirow{2}{*}{ MSE } & \multirow{2}{*}{ TD } \\
\hline & $\gamma$ & $\beta$ & $\hat{\gamma}$ & $\hat{\beta}$ & & \\
\hline \multirow{3}{*}{ LSM } & 1 & 1 & 0.999996 & 0.999924 & $2.041 \times 10^{-8}$ & 0.0000789 \\
\hline & 1 & 2 & 1.004568 & 1.99696 & 0.0000482 & 0.0060837 \\
\hline & 2 & 3 & 2.00000185 & 2.99999 & $4.1 \times 10^{-12}$ & 0.0000012 \\
\hline \multirow{3}{*}{ RELS } & 1 & 1 & 0.99999 & 0.99999746 & $4.0544 \times 10^{-11}$ & 0.0000032 \\
\hline & 1 & 2 & 0.99992 & 2.0227 & 0.0003941 & 0.0113576 \\
\hline & 2 & 3 & 2.0001945 & 2.999844 & $3.567 \times 10^{-8}$ & 0.0001493 \\
\hline \multirow{3}{*}{ RR } & 1 & 1 & 0.992288 & 0.994180 & 0.0013807 & 0.013532 \\
\hline & 1 & 2 & 1.01501 & 1.96564 & 0.0004904 & 0.03219 \\
\hline & 2 & 3 & 2.00730 & 2.95982 & 0.0003316 & 0.0170433 \\
\hline \multirow{3}{*}{ ME } & 1 & 1 & 1.15174 & 0.654301 & 0.414524 & 0.497439 \\
\hline & 1 & 2 & 1.30347 & 1.30860 & 0.414503 & 0.64917 \\
\hline & 2 & 3 & 2.45521 & 1.962901 & 0.414511 & 0.573305 \\
\hline \multirow{3}{*}{ (MME-I) } & 1 & 1 & 0.982780 & 0.823257 & 0.157346 & 0.193965 \\
\hline & 1 & 2 & 0.965560 & 1.646513 & 0.157347 & 0.211184 \\
\hline & 2 & 3 & 1.94834 & 2.46977 & 0.157347 & 0.202573 \\
\hline \multirow{3}{*}{ (MME-II) } & 1 & 1 & 0.982520 & 0.823516 & 0.157587 & 0.193964 \\
\hline & 1 & 2 & 0.965040 & 1.647032 & 0.157587 & 0.211444 \\
\hline & 2 & 3 & 1.947560 & 2.470548 & 0.157857 & 0.202704 \\
\hline \multirow{3}{*}{ (MME-III) } & 1 & 1 & 1.00950 & 0.796538 & 0.138321 & 0.212962 \\
\hline & 1 & 2 & 1.01900 & 1.59308 & 0.138317 & 0.22246 \\
\hline & 2 & 3 & 2.02849 & 2.38962 & 0.138324 & 0.217705 \\
\hline \multirow{3}{*}{ MLE } & 1 & 1 & 1.00311 & 0.802928 & 0.141786 & 0.200182 \\
\hline & 1 & 2 & 1.00622 & 1.60586 & 0.141783 & 0.20329 \\
\hline & 2 & 3 & 2.00932 & 2.40878 & 0.141796 & 0.201733 \\
\hline \multirow{3}{*}{ MMLE-I } & 1 & 1 & 0.983035 & 0.802928 & 0.193612 & 0.214037 \\
\hline & 1 & 2 & 0.966070 & 1.60586 & 0.193608 & 0.231 \\
\hline & 2 & 3 & 1.94911 & 2.40878 & 0.193610 & 0.222518 \\
\hline
\end{tabular}


Table 3: The estimates for $n=60$

\begin{tabular}{|c|c|c|c|c|c|c|}
\hline \multirow{2}{*}{ Method } & \multicolumn{2}{|c|}{ True Values } & \multicolumn{2}{|c|}{ Estimated Values } & \multirow{2}{*}{ MSE } & \multirow{2}{*}{ TD } \\
\hline & $\gamma$ & $\beta$ & $\hat{\gamma}$ & $\hat{\beta}$ & & \\
\hline \multirow{3}{*}{ LSM } & 1 & 1 & 0.9999982 & 0.9999975 & $6.5 \times 10^{-10}$ & 0.0000094 \\
\hline & 1 & 2 & 1.00005263 & 2.0004348 & 0.0000003 & 0.00027 \\
\hline & 2 & 3 & 2.0000054 & 2.999996 & $3.66 \times 10^{-11}$ & 1.0000016 \\
\hline \multirow{3}{*}{ RELS } & 1 & 1 & 1.0000094 & 1.0000038 & $2.1 \times 10^{-9}$ & 0.0000132 \\
\hline & 1 & 2 & 1.0000174 & 1.999886 & $9.68 \times 10^{-9}$ & 0.0000741 \\
\hline & 2 & 3 & 1.999963 & 2.99995 & $6.4 \times 10^{-9}$ & 0.000034 \\
\hline \multirow{3}{*}{ RR } & 1 & 1 & 0.990869 & 1.00078 & 0.0013624 & 0.009911 \\
\hline & 1 & 2 & 0.998391 & 1.99403 & 0.0000859 & 0.004594 \\
\hline & 2 & 3 & 1.98926 & 2.99481 & 0.0003236 & 0.0071 \\
\hline \multirow{3}{*}{ ME } & 1 & 1 & 1.01216 & 1.11234 & 0.0719734 & 0.1245 \\
\hline & 1 & 2 & 1.02432 & 2.22468 & 0.0719734 & 0.13666 \\
\hline & 2 & 3 & 2.03648 & 3.337022 & 0.0719741 & 0.130581 \\
\hline \multirow{3}{*}{ (MME-I) } & 1 & 1 & 0.996175 & 1.12833 & 0.0623616 & 0.132155 \\
\hline & 1 & 2 & 0.992349 & 2.25665 & 0.0623561 & 0.135976 \\
\hline & 2 & 3 & 1.988523 & 3.384975 & 0.0623559 & 0.134063 \\
\hline \multirow{3}{*}{ (MME-II) } & 1 & 1 & 0.996017 & 1.12848 & 0.0622806 & 0.132463 \\
\hline & 1 & 2 & 0.992034 & 2.25697 & 0.0622854 & 0.136451 \\
\hline & 2 & 3 & 1.988051 & 3.385448 & 0.0622831 & 0.134457 \\
\hline \multirow{3}{*}{ (MME-III) } & 1 & 1 & 1.04236 & 1.08214 & 0.103420 & 0.1245 \\
\hline & 1 & 2 & 1.08471 & 2.16429 & 0.103414 & 0.166855 \\
\hline & 2 & 3 & 2.12707 & 3.24643 & 0.103416 & 0.145678 \\
\hline \multirow{3}{*}{ MLE } & 1 & 1 & 1.01483 & 1.10967 & 0.0740230 & 0.1245 \\
\hline & 1 & 2 & 1.02965 & 2.219349 & 0.0740185 & 0.139324 \\
\hline & 2 & 3 & 2.04448 & 3.3290 & 0.0740204 & 0.131913 \\
\hline \multirow{3}{*}{ MMLE-I } & 1 & 1 & 0.996330 & 1.10967 & 0.0458705 & 0.11334 \\
\hline & 1 & 2 & 0.992660 & 2.219349 & 0.0458743 & 0.117014 \\
\hline & 2 & 3 & 1.98899 & 3.32902 & 0.0458733 & 0.115178 \\
\hline
\end{tabular}


Table 4: The estimates for $n=80$

\begin{tabular}{|c|c|c|c|c|c|c|}
\hline \multirow{2}{*}{ Method } & \multicolumn{2}{|c|}{$\begin{array}{c}\text { True } \\
\text { values }\end{array}$} & \multicolumn{2}{|c|}{ Estimated Values } & \multirow{2}{*}{ MSE } & \multirow{2}{*}{ TD } \\
\hline & $\gamma$ & $\beta$ & $\hat{\gamma}$ & $\hat{\beta}$ & & \\
\hline \multirow{3}{*}{ LSM } & 1 & 1 & 0.999998 & 0.9999977 & $1.84312 \times 10^{-10}$ & 0.0000039 \\
\hline & 1 & 2 & 0.99999802 & 1.999998 & $4.0589 \times 10^{-11}$ & 0.0000025 \\
\hline & 2 & 3 & 1.9999955 & 2.999996 & $1.14738 \times 10^{-10}$ & 0.0000036 \\
\hline \multirow{3}{*}{ RELS } & 1 & 1 & 1.0000176 & 1.0000132 & 0.0000001 & 0.0000898 \\
\hline & 1 & 2 & 1.0000082 & 2.0000144 & $1.355 \times 10^{-9}$ & 0.0000153 \\
\hline & 2 & 3 & 1.999994 & 2.99997 & $7.9446 \times 10^{-10}$ & 0.0000109 \\
\hline \multirow{3}{*}{$\mathrm{RR}$} & 1 & 1 & 0.995201 & 0.998303 & 0.0008376 & 0.006496 \\
\hline & 1 & 2 & 1.00363 & 1.98818 & 0.0001032 & 0.00954 \\
\hline & 2 & 3 & 1.99883 & 2.98649 & 0.0001617 & 0.0050883 \\
\hline \multirow{3}{*}{$\mathrm{ME}$} & 1 & 1 & 1.053605 & 0.783958 & 0.193896 & 0.269647 \\
\hline & 1 & 2 & 1.0721 & 1.56792 & 0.223028 & 0.28814 \\
\hline & 2 & 3 & 2.16082 & 2.35187 & 0.193897 & 0.296453 \\
\hline \multirow{3}{*}{ (MME-I) } & 1 & 1 & 0.989691 & 0.847872 & 0.205316 & 0.162437 \\
\hline & 1 & 2 & 0.979383 & 1.69574 & 0.205309 & 0.172742 \\
\hline & 2 & 3 & 1.96907 & 2.54362 & 0.205317 & 0.167592 \\
\hline \multirow{3}{*}{ (MME-II) } & 1 & 1 & 0.989625 & 0.847938 & 0.205412 & 0.162437 \\
\hline & 1 & 2 & 0.979250 & 1.69588 & 0.2054069 & 0.17281 \\
\hline & 2 & 3 & 1.96887 & 2.54382 & 0.205414 & 0.167625 \\
\hline \multirow{3}{*}{ (MME-III) } & 1 & 1 & 0.959586 & 0.877977 & 0.262005 & 0.162437 \\
\hline & 1 & 2 & 0.919172 & 1.75595 & 0.262011 & 0.202583 \\
\hline & 2 & 3 & 1.87876 & 2.63393 & 0.2620024 & 0.182643 \\
\hline \multirow{3}{*}{ MLE } & 1 & 1 & 1.00022 & 0.837339 & 0.191808 & 0.162881 \\
\hline & 1 & 2 & 1.00045 & 1.67468 & 0.191787 & 0.16311 \\
\hline & 2 & 3 & 2.00067 & 2.51202 & 0.191792 & 0.162995 \\
\hline \multirow{3}{*}{ MMLE-I } & 1 & 1 & 0.989757 & 0.837339 & 0.234187 & 0.172904 \\
\hline & 1 & 2 & 0.979514 & 1.67468 & 0.234184 & 0.183146 \\
\hline & 2 & 3 & 1.969271 & 2.51202 & 0.234184 & 0.178024 \\
\hline
\end{tabular}


Table 5: The estimates for $n=100$

\begin{tabular}{|c|c|c|c|c|c|c|}
\hline \multirow{2}{*}{ Method } & \multicolumn{2}{|c|}{ True Values } & \multicolumn{2}{|c|}{ Estimated Values } & \multirow{2}{*}{ MSE } & \multirow{2}{*}{ TD } \\
\hline & $\gamma$ & $\beta$ & $\hat{\gamma}$ & $\hat{\beta}$ & & \\
\hline \multirow{3}{*}{ LSM } & 1 & 1 & 1.00000202 & 1.0000017 & $2.58 \times 10^{-10}$ & 0.0000037 \\
\hline & 1 & 2 & 1.00000213 & 2.0000022 & $7.884 \times 10^{-11}$ & 0.0000032 \\
\hline & 2 & 3 & 2.00000312 & 2.999967 & $6.105 \times 10^{-10}$ & 0.0000127 \\
\hline \multirow{3}{*}{ RELS } & 1 & 1 & 1.00000113 & 1.00000111 & $8.72 \times 10^{-11}$ & 1.000002 \\
\hline & 1 & 2 & 0.9999394 & 2.0000044 & $3.52 \times 10^{-8}$ & 0.000067 \\
\hline & 2 & 3 & 2.0000157 & 2.999997 & $9.81 \times 10^{-10}$ & 0.0000089 \\
\hline \multirow{3}{*}{$R R$} & 1 & 1 & 0.995823 & 0.999050 & 0.0007896 & 0.005127 \\
\hline & 1 & 2 & 1.00085 & 1.99307 & 0.0000546 & 0.004315 \\
\hline & 2 & 3 & 1.99667 & 2.992125 & 0.0001602 & 0.00429 \\
\hline \multirow{3}{*}{ ME } & 1 & 1 & 0.930781 & 0.914596 & 0.394559 & 0.154623 \\
\hline & 1 & 2 & 0.861562 & 1.82919 & 0.394562 & 0.223843 \\
\hline & 2 & 3 & 1.79234 & 2.74379 & 0.394564 & 0.189233 \\
\hline \multirow{3}{*}{ (MME-I) } & 1 & 1 & 0.995422 & 0.849955 & 0.196354 & 0.15458 \\
\hline & 1 & 2 & 0.990844 & 1.69991 & 0.196344 & 0.159201 \\
\hline & 2 & 3 & 1.98627 & 2.54987 & 0.196344 & 0.156908 \\
\hline \multirow{3}{*}{ (MME-II) } & 1 & 1 & 0.995380 & 0.849998 & 0.196423 & 0.154622 \\
\hline & 1 & 2 & 0.990760 & 1.699995 & 0.196424 & 0.159243 \\
\hline & 2 & 3 & 1.98614 & 2.54999 & 0.196426 & 0.156933 \\
\hline \multirow{3}{*}{ (MME-III) } & 1 & 1 & 0.6126 & 1.23278 & 2.18395 & 0.62018 \\
\hline & 1 & 2 & 0.225200 & 2.46555 & 2.18398 & 1.00758 \\
\hline & 2 & 3 & 0.837800 & 3.69833 & 2.18397 & 0.813877 \\
\hline \multirow{3}{*}{ MLE } & 1 & 1 & 1.00388 & 0.841498 & 0.184035 & 0.162382 \\
\hline & 1 & 2 & 1.00776 & 1.68300 & 0.184029 & 0.16626 \\
\hline & 2 & 3 & 2.01164 & 2.52449 & 0.184038 & 0.164323 \\
\hline \multirow{3}{*}{ MMLE-I } & 1 & 1 & 0.995465 & 0.841498 & 0.219908 & 0.163037 \\
\hline & 1 & 2 & 0.990930 & 1.68300 & 0.219903 & 0.16757 \\
\hline & 2 & 3 & 1.98639 & 2.52449 & 0.219921 & 0.165308 \\
\hline
\end{tabular}




\section{Conclusion}

The results are listed in the tables (1), (2), (3), (4) and (5). From the computations, we note that, the estimates of parameters from the LSM are too close to the true values, and the values of MSE and TD are very small. The parameter estimates from RELS, RR method are close to the true values but not as LS estimates, because the values of MSE and TD are greater than the corresponding values from LSM.

In this article, our proposed third modified moment estimators (MME-III) is given better accuracy as compared to previously defined traditional method of moments (ME) and first modified moment estimators (MME-I) for small samples of size $n=20,40$. For the samples of large size $n=100$, the values of MSE and TD obtained from (MME-111) are too large.

Another modification which we made in this article is the first modified maximum likelihood estimators (MMLE-I). First modified maximum likelihood estimators (MMLE-I) is given better accurate estimates as compared to previously defined traditional method of moments (ME) and maximum likelihood method for the samples of size $n=20,60$, because they gave the least value for the mean square error.

\section{References}

1. Cohen, A.C., and Helm, F.R. (1973). Estimation in Exponential Distribution. Technometrics 15, 415-418.

2. Cohen, A.C. and Whitten, B.J. (1982b). Modified Maximum Likelihood and Moment Estimation of three Parameters Weibull Distribution. Commun. Statist. Theory and Method 11(23), 2631-2656.

3. Evans, M., Hasting, N.A.J. and Peacocks, J.B. (2000). Statistical Distribution ( $3^{r d}$ ed.). Wiley Series in Probability and Statistic, New York.

4. Kang, S.B. and Young, C.S. (1997). Estimation of the Parameters in a Pareto Distribution by Jackknife and Bootstrap Methods. Journal of Information and Optimization Sci. 18, 289-300.

5. Keceioglu, D. (1991). Reliability Engineering Handbook. Prentice Hall, Eaglewood Cliffs, NJ.

6. Lalitha, S. and Anand, M. (1996). Modified Maximum Likelihood Estimators for Rayleigh Distribution. Commun. Statist., Theory and Method 25 (2), 389-401.

7. Maguire, B.A., Pearson, E.S. and Wynn, A.H.A. (1952). The time intervals between industrial accidents. Biometrika 39, 168-180.

8. Mohammed A. AL-Fawzan (2000). Algorithms for Estimating the Parameters of the Weibull Distribution, King Abdul-Aziz City for Science and Technology, Riyadh, Saudi Arabia. 
9. Pablo, B.S. and Bruce, E.R. (1992). Model Parameter Estimation using Least Squares. Water Res. 26(6), 789-796.

10. Peter, F. Thall, (1974). Huber-Sense Robust M-Estimation of a Scale Parameter, with Application to the Exponential Distribution. JASA74, 147-152.

11. Ronald, E.W. and Raymond, H.M. (1978). Probability and Statistics for Engineers and Scientists ( $2^{\text {nd }}$ ed). McMillan Publishing Co., Inc., New York.

12. Samia, A.S. and Mohammed M.M. (1993). Modified Moment Estimators for three parameters Pareto distribution. ISSR, Cairo University 28 (2).

13. Sidney, Y. and Ferenc, S. (1989). An Introduction to Numerical Computations ( $2^{\text {nd }}$ ed.). Macmillan Publishing Company, New York.

14. Yidirim, F. (1990). Least Squares Estimation for the Weibull Parameters, Pakistan Journal of Statistics, 6(1) A, 93-104. 